\title{
Third Greatest Diameter
}

National Cancer Institute

\section{Source}

National Cancer Institute. Third Greatest Diameter. NCI Thesaurus. Code C101709.

The third longest possible length of a straight line passing through the center of a nearly circular or spheroid object that connects two points on the circumference. 